\title{
Complicaciones del divertículo de Meckel. Análisis clínico e histopatológico de 11 casos
}

DRES.: FRANCISCO LARRAIN ", USVALDO DANUS *.

El conducto onfalomesentérico que comunica el ileon con el saco amniótico, desaparece habitualmente despućs de la sexta semana de vida fetal. Puede persistir sin embargo, en algunos casos, dando origen a diversas anomalías, siendo la más frecuente de ellas el divertículo de Meckel. Este se forma en el 2 a $3 \%$ de los individuos y está ubicado en el jleon terminal, en el lado opuesto al mesenterio, a poca distancia de la válvula ileocecal (60-90 cm.) (1).

La presencia de tejido heterotópico en su interior y la fijación de su extremo libre por medio de bandas fibrosas o adherencias inflamatorias, pueden producir numerosas complicaciones, como son la hemorragia, diverticulitis, perforación u obstrucción intestinal, las que conducen a cuadros clínicos graves, con síntomas poco específicos en los cuales ni la clínica ni el estudio radiológico prestan ayuda suficiente para esclarecer el diagnóstico. Aunque relativamente poco frecuentes los casos complicados son de tal gravedad, que justifican las numerosas publicaciones aparecidas acerca de esta malformación.

En la presente publicación, se analizan los casos ocurridos en este hospital en un período de 8 años.

Matertal y método. En esta revisión se comentan los aspectos clinicos, hallazgos quirúrgicos y/o de necropsia, de 17 niños que acudieron a este hospital entre los años 1966 y 1973 y presentaban un divertículo de Meckel.

Once de los 17 casos, ingresaron por síntomas debidos a una complicación del divertículo de Meckel. En todos ellos el diagnóstico se hizo mediante la intervención quirúrgica. En los 6 casos restantes el diagnóstico constituyó un hallazgo de autopsia, en niños fallecidos por otra enfermedad.

- Gastroenccrología.

* Departamento de Pediatría, Hospital Roberto del Ro.
Existió un franco predominio del sexo masculino sobre el femenino, en una proporción de 4:1 (14 hombres y 3 mujeres).

$\mathrm{La}$ edad de los pacientes vario entre 1 día $\mathrm{y}$ 7 años, pero la gran mayoría de los niños (14 pacientes) tenía una edad inferior a los 2 años. Los 3 casos restantes tenían 6 años ( 2 casos) y 7 años de eđad ( 1 caso). Se analizó detenidamente la historia clínica de los niños con divertículo de Meckel complicado, para precisar la sintomatología, los hallazgos en el examen de ingreso, la cifra de hemoglobina (cuando hubo rectorragias) el diaguóstico planteado previo al acto quirúrgico y la evolución post operatoria. Se analizaron además los hallazgos del cirujano y del anátomopatologo, para precisar el tipo de complicación presentada. En 1 caso diagnosticado por la autopsia existía una cardiopatía congénita asociada.

ResultaDos. De acuerdo al modo de presentación los casos sintomáticos pueden ser separados en dos grupos: 1) Aquellos niños que ingresaron por un cuadro de abdomen agudo (9 casos) y 2) los que presentaron hemorragia rectal (2 pacientes).

La forma de presentación está esquematizada fuc la siguiente:

FORMA DE PRESENTACION DE 17 CASOS DE DIVERTICULO DE MECKEL

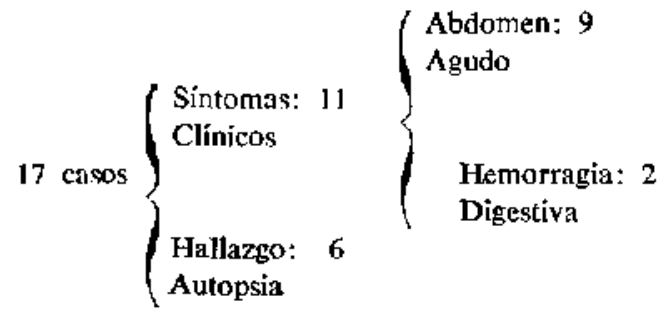




\section{1) Abdomen Agudo}

Los dates clínicos más importantes de este givo de niños, están resumidos en la Tabla No 1 . En todos ellos existió una historia clínica breve (de 1 a 3 cías de evolución) caracterizada por dolor abdiminal de intensidad variabie, en algunos casos de tipo cólico y vómitos persistentes, a' principio ce contenido alimentario y luego bjfiosos. Ln 3 casos existieron vómitos de retención. Cinco ninos tuvicron además fiebre.

En el examen físico de ingreso se encontró el abdomen distendido y sensible en todos los pacientes, con resistencia nuscular y Bloomberg esbozado cn 4 casos. In 1 paciente se palpaba una gran misa abdominal muy sensible, que ocupuba casi trdo el hemiabdomen derecho. El diagnóstico de ingreso fue de sepsis on 4 casos, obstrucción intestinal en 2 , invaginación intestinal en 1 y apendicitis aguda en 1 caso. En el niño de la masa abjominal se planteó el diagnóstico de riñón poliquístico.

Cuitro de los pacicntes fueron intervenidos. Los hailazgos operatorios y el estudio histopatoló- gico, demostraron una obstrucción intestinal prodecicta por el diverticulo de Meckel, en 3 casos. En 2 de ellos el cirujano describe la presencia de una banda fibrosa, que unía el divertículo a la pared abdominal y que era la responsable de la obstrucción. En el cuarto caso se comprobó una porforación del diverticulo y una peritonitis secundaria. A todos cstos pacientes se les practicó una resección intestinal y la evolución posterior fue satisfactoria, siendo datos de alta entre los 7 y los 15 días después, en muy buenas condiciones.

Cinco niños fallecieron a las pocas horas del ingreso. Ninguno de ellos fue intervenido; en cuatro de el'os, el estudio anátomopatologico demostró una diverticulitis primaria, sin obstrucción mecánic:t. En el quinto caso, existía una obstrucción intestinal con compromiso vascular y peritonitis secundaria.

\section{2) Hemorragia rectal}

En Ja tabla No 2 están resumidos los hechos c.inicos más relevantes de los 2 pacientes que consultaron por hemorragia rectal. Los 2 cran lac-

TABLA No 1

DIERTKULO DE MECKEL. CARACTERISTKAS EN 9 CASOS CON ABDOMEN AGUDO

\begin{tabular}{|c|c|c|c|c|c|c|c|}
\hline Edad & Evol. Enf. & Sintomas & Exumen ingreso & $\begin{array}{l}\text { Diagnóst. pre } \\
\text { operutorio }\end{array}$ & $\begin{array}{l}\text { Hallazjos } \\
\text { quirúgicos }\end{array}$ & $\begin{array}{l}\text { Anátomo } \\
\text { patologicos }\end{array}$ & Evolución \\
\hline 7 años & 2 días & $\begin{array}{l}\text { Dolor } \\
\text { abdominal } \\
\text { Vómitos }\end{array}$ & $\begin{array}{l}\text { Disten. Abdominal } \\
\text { Resist. muscular } \\
\text { Bloomberg }\end{array}$ & $\begin{array}{l}\text { Obstrucción } \\
\text { intestinal }\end{array}$ & $\begin{array}{l}\text { Obstrucción } \\
\text { intestinal }\end{array}$ & - & Buena \\
\hline I a. 8 nIs. & 3 datas & $\begin{array}{l}\text { Dolor } \\
\text { abdominal } \\
\text { Vómitos } \\
\text { Fiebre }\end{array}$ & $\begin{array}{l}\text { Disten. Abdominal } \\
\text { Defensa muscular } \\
\text { Bloomberg }\end{array}$ & $\begin{array}{l}\text { Invagina- } \\
\text { ción } \\
\text { Intestinal }\end{array}$ & $\begin{array}{l}\text { Obstrucción } \\
\text { intestinal }\end{array}$ & - & Buena \\
\hline 6 :ลn๋๐ & 1 dia & $\begin{array}{l}\text { Dolor } \\
\text { abdominal } \\
\text { Vómitos } \\
\text { Fiebre }\end{array}$ & $\begin{array}{l}\text { Disten. Abdominal } \\
\text { Bloomberg }\end{array}$ & $\begin{array}{l}\text { Apendicitis } \\
\text { aguda }\end{array}$ & $\begin{array}{l}\text { Diverticulitis } \\
\text { Perforación }\end{array}$ & 一 & Buena \\
\hline 6 años & I dia & $\begin{array}{l}\text { Dolor } \\
\text { abdominal } \\
\text { Vómitos }\end{array}$ & $\begin{array}{l}\text { Disten. Abdominal } \\
\text { Defensa muscular } \\
\text { B loomberg }\end{array}$ & $\begin{array}{l}\text { Obstrucción } \\
\text { intestinal }\end{array}$ & $\begin{array}{l}\text { Obstrucción } \\
\text { intestinal }\end{array}$ & - & Buena \\
\hline $1 \mathrm{a} .6 \mathrm{~ms}$ & 1 día & $\begin{array}{l}\text { Vómitos } \\
\text { Decaimiento } \\
\text { Fiebre }\end{array}$ & $\begin{array}{l}\text { Disten. Abdominal } \\
\text { Defensa museular } \\
\text { Hiperperistalsis }\end{array}$ & Sepsis & $\cdots$ & $\begin{array}{l}\text { Obstrucción } \\
\text { intestinal } \\
\text { Peritonitis }\end{array}$ & Fallece \\
\hline 7 meses & 2 dias & $\begin{array}{l}\text { Fiebre } \\
\text { Vómitos }\end{array}$ & $\begin{array}{l}\text { Disten. Abdominal } \\
\text { Deshidratación }\end{array}$ & Sepsis & - & Diverticulitis & Fallece \\
\hline 1 dìi & $\begin{array}{l}\text { Desde } \\
\text { nacimiento }\end{array}$ & $\begin{array}{l}\text { Masa } \\
\text { palpable } \\
\text { Vómitos }\end{array}$ & Masa abdominal & $\begin{array}{l}\text { Riñón } \\
\text { Poliquístico }\end{array}$ & - & $\begin{array}{l}\text { Diverticulitis } \\
\text { Peritonitis }\end{array}$ & Fallcce \\
\hline 8 meses & 2 días & $\begin{array}{l}\text { Fiebre } \\
\text { Vómitos }\end{array}$ & $\begin{array}{l}\text { Disten. Abdominal } \\
\text { Hiperperistalsis }\end{array}$ & $\begin{array}{l}\text { Sepsis } \\
\text { Vomitador }\end{array}$ & - & Diverticulitis & Fallece \\
\hline $1 \mathrm{~m} .7 \mathrm{ds}$ & 1 día & $\begin{array}{l}\text { Vómitos } \\
\text { Deshidra- } \\
\text { tación. }\end{array}$ & $\begin{array}{l}\text { Disten. Abdominal } \\
\text { Silencio Abdo- } \\
\text { minal }\end{array}$ & Sepsis & 一 & Diverticulitis & Fallece \\
\hline
\end{tabular}


T A B L A Nọ 2

DIVERTICULO DE MECKEL. CARACTERISTICAS EN 2 CASOS CON HEMORRAGIA RECTAL

\begin{tabular}{|c|c|c|c|c|c|c|}
\hline Edad & Evol. Enf. & Henorragia Rectal & Ex Ingreso & Hb. Ingreso & Anat. Patol. & Evolucion \\
\hline 1 a. $8 \mathrm{~m}$. & 1 día & $\begin{array}{l}\text { Masiva. Sangre } \\
\text { negra y roja }\end{array}$ & $\begin{array}{c}\text { Palidez } \\
\text { Taquic. 180, }\end{array}$ & $5 \mathrm{~g} \%$ & $\begin{array}{c}\text { Mucosa Gas. } \\
\text { ulcerada }\end{array}$ & Buena \\
\hline I a. $5 \mathrm{~m}$, & 1 año & $\begin{array}{l}\text { Mezcla de sangre } \\
\text { negra y roja }\end{array}$ & $\begin{array}{c}\text { Palidez } \\
\text { Taquic, } 160^{\circ}\end{array}$ & $5,2 \mathrm{~g} \%$ & $\begin{array}{c}\text { Mucosa Gas. } \\
\text { ulcerada }\end{array}$ & Buena \\
\hline
\end{tabular}

tantes mayores. Uno de ellos tenía el antecedente de haber presentado episodios anteriores de rectorragia (seis episodios de hemorragia digestiva en un año). El otro presentaba hemorragia rectal por primera vez. En ambos casos la hemorragia fue intensa, llegándose a producir un cuadro de anemia aguda (palidez intensa, taquicardia de 150-180 x', hemoglobinas de 5 y 5,2 gramos \%). La sangre eliminada tenia las características de hemotoquexia, es decir mezcla de melena y rectorragia. También en ambos casos la pared del divertículo estaba tapizada de mucosa gástrica ulcerada y sangrante. Los pacientes fueron intervenidos y la evolución postoperatoria fue excelente.

Comentario. Del análisis del material revisado, destaca el hecho que solamente 2 niños (18\% de los casos complicados), consultaron por hemorragia rectal. Esto contrasta con lo descrito en otras publicaciones, en las cuales la hemorragia digestiva constituye un síntoma fundamental en un alto porcentaje de los casos (2). Así por ejemplo, en una revisión hecha por Rutherford y Akers (3) que comprende 80 casos de divertículo de Meckel complicados, puede verse que el 53\% de ellos consultaron por hemorragia digestiva. La baja incidencia que presenta la hemorragia digestiva en nuestra serie, puede ser atribuída al hecho que los casos que se presentan como abdomen agudo obligan a la intervención inmediata. En cambio, tenemos la impresión de que en el grupo de nuestros pacientes que están en control por hemorragia digestiva de causa aún no precisada, deben existir algunos niños portadores de un divertículo de Meckel.

En nuestros 2 casos, la hemorragia tuvo características similares a las descritas por los autores señalados, en relación a su cuantía y a los ballazgos anátomopatologicos. En efecto, en ambos la hemorragia fue intensa, llevó a los pacientes a un estado de anemia aguda con shock y la hemoglobina de ingreso fue inferior a 8 gramos\%. En ambos casos también, el estudio histopatolo gico reveló la presencia de mucosa gástrica heterotópica, con una úlcera sangrante en su interior. La sangre eliminada tuvo las características de hematoquexia; aunque este tipo de sangramiento se describe con cierta frecuencia en la hemorragia del divertículo de Meckel, no sjempre está presente, ya que en algunos casos pueden existir rectorragia o melena por separado (3).

La mayor parte de los pacientes $(9$ de los 11 casos), ingresó al hospital por un cuadro abdominal agudo, caracterizado por vómitos, dolor y distensión abdominal, con signos de irritación peritoneal en la mitad de ellos. La mayoría de estos niños eran lactantes. Creemos que cuando se está frente a un cuadro abdominal agudo en un niño, especialmente si se trata de un lactante, debe tenerse presente esta posibilidad etiológica.

\section{RESUMEN}

Se analiza la evolución clinica y los hallazgos anátomopatologicos de 11 pacientes que presentaron un diverticulo de Meckel complicado. La mayoría de los niños eran lactantes. El $82 \%$ de ellos consultó por un cuadro abdominal agudo, producido por diverticulitis (4 casos), obstrucción intestinal ( 4 casos) y perforación del diverticulo (1 caso). Sólo 2 niños tuvieron hemorragia rectal, la que fue intensa con características de hematoquexia. En ambos, el sangramiento se debio a la presencia de mucosa gastrica heterotopica ulcerada en el interior del diverículo.

\section{SUMMARY}

The clinical evolution as well as the pathologic findings in 11 patients presenting a complicated Mec. kel's Diverticulum, are discused. Most patients were infants. An acute abdominal pain was present in $82 \%$ of the cases, corresponding to diverticulitis (4 cases), intestinal obstruction (4 cases), and perforatin of the diverculum ( 1 case). Only two patients evidenced rectal bleeding which was profuse. In both cases there was gastric mucosa with peptic ulceration in the Meckel's Diverticulum.

\section{REFERENCIAS}

1.- Nelson, W. E. et Al. Tratado de Pediatría. Ed. Barcelona, Salvat, 1971.

2.-Silverman A., Roy, C. Pediatric Clinical Gastroenterology St. Louis, The C. V. Mosby Co., 1971.

3.-Rutherford, R. B., and Akers, D. R. Meckel's diverticulum Surgery 59: 618, 1966. 